\title{
Auf Leben und Tod - Steigende Lebenserwartung und Sozialversicherung*
}

\author{
Friedrich Breyer \\ Universität Konstanz und DIW Berlin
}

\section{Einleitung}

Einer der Faktoren, der in der Zukunft zu ständig steigenden Beitragssätzen in der gesetzlichen Rentenversicherung führen wird, ist die wachsende Langlebigkeit der Deutschen, statistisch erfasst in der (fernen) Lebenserwartung der 65jährigen. Konnte eine 65jährige Frau im Jahre 1950 im Durchschnitt noch mit 13,7 weiteren Lebensjahren rechnen, so sind es heute (1999) bereits 19,2 Jahre, und der Trend ist ungebrochen. Die Konsequenzen sind dramatisch steigende Rentenlaufzeiten und daher eine wachsende Zahl von Rentnern pro Kopf der Erwerbsbevölkerung, ganz unabhängig von der schrumpfenden Kohortengröße auf Grund der seit 1970 rückläufigen Geburtenzahlen. Bemerkenswert an diesem Phänomen ist, dass es die Finanzierung der Renten in jedem Fall erschwert, unabhängig davon, ob diese im Umlageverfahren oder via Kapitaldeckung finanziert werden.

Dass die ferne Lebenserwartung der 65jährigen steigt, ist im übrigen weder ein neues noch auf Deutschland beschränktes Phänomen. Tabelle 1 enthält Daten zur Entwicklung der Lebenserwartung für 65jährige Männer und Frauen in allen OECD-Ländern seit 1970. Aus den Zahlen wird deutlich, dass diese in den letzten drei Jahrzehnten nicht nur recht kräftig gestiegen ist, nämlich im Durchschnitt um 2,63 Jahre bei Männern und 3,27 Jahre bei Frauen. Darüber hinaus hat sich das Tempo des Anstiegs nicht vermindert, was man erkennt, wenn man berücksichtigt, dass der letzte der drei Vergleichszeiträume nur 9 statt 10 Jahren umfasst. Bei den Frauen lässt sich dabei ein gewisser "Aufholeffekt" in dem Sinne beobachten, dass die Lebenserwartung seit 1990 in den Ländern stärker gestiegen ist, in denen der Ausgangswert niedriger lag: der Korrelationskoeffizient zwischen beiden

\footnotetext{
*Der Autor dankt Martin Heineck, Normann Lorenz, Christine Holzem und Andrej Grimm für Unterstützung bei der empirischen Datenanalyse sowie Mathias Kifmann, Martin Kolmar, den Teilnehmern der Jahrestagung des Ausschusses für Bevölkerungsökonomie 2003 in Berlin und einem anonymen Referee für fruchtbare Diskussionen und wertvolle Hinweise.
}

(C) Verein für Socialpolitik und Blackwell Publishing Ltd. 2004, 9600 Garsington Road, Oxford OX4 2DQ, UK und 350 Main Street, Malden, MA 02148, USA. 


\section{Friedrich Breyer}

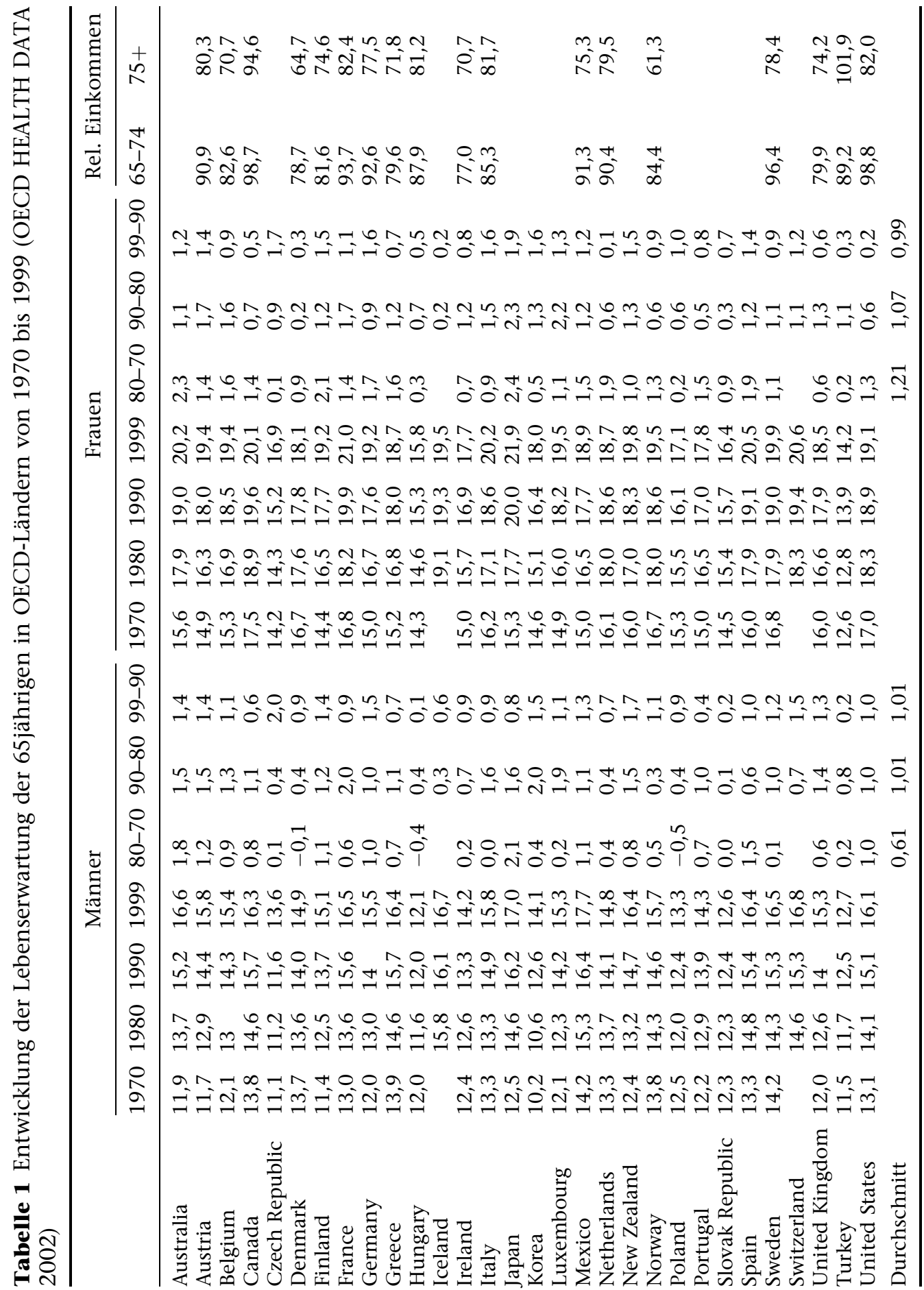




\section{Auf Leben und Tod}

Reihen beträgt $-0,4{ }^{1}$ Bei den Männern ist der entsprechende Koeffizient $(+0,08)$ nicht signifikant von null verschieden.

Während die Gefährdung der Rentenfinanzierung durch die steigende Lebenserwartung heute schon gut erkannt ist und vielfach diskutiert wird, gibt es lediglich eine kleine Anzahl noch wenig beachteter Arbeiten von Ökonomen, die sich mit der These befassen, dass auch ein umgekehrter Zusammenhang bestehen könnte: ${ }^{2}$ Möglicherweise tragen gerade die Sozialversicherungen zu der massiven Verlängerung der Lebenserwartung bei und verschärfen damit das Problem für ihr eigenes finanzielles Überleben. Der vorliegende Beitrag soll auf diese Arbeiten aufmerksam machen und eine Diskussion darüber anstoßen, welche institutionellen Reformen den behaupteten Mechanismus stoppen und damit die Nachhaltigkeit der Sozialsysteme sichern können.

Strukturell existiert eine Ähnlichkeit zwischen dem hier zu untersuchenden Zusammenhang und der vor gut einem Jahrzehnt von Zweifel (1990) aufgestellten These vom Bestehen eines Sisyphos-Syndroms in der sozialen Krankenversicherung. Diese behauptet, dass mit den Fortschritten in der Medizin die Lebenserwartung steigt und damit der Anteil der Älteren in der (wahlberechtigten) Bevölkerung zunimmt. Die Älteren wiederum können im politischen Prozess eine Erhöhung der Ausgaben für medizinische Forschung und Behandlung durchsetzen, womit der Trend zur Erhöhung der Lebenserwartung verstärkt wird. Der zusätzliche Aspekt, der von den hier zu referierenden Arbeiten in die Debatte eingebracht wird, betrifft das Interesse der Menschen an einer höheren Lebenserwartung und erklärt dieses mit der großzügigen finanziellen Versorgung im Alter durch (staatliche oder private) Leibrenten.

Der Beitrag ist wie folgt aufgebaut: In Abschnitt 2 werden die theoretischen Modelle von Davies und Kuhn (1992) sowie Philipson und Becker (1998) rekapituliert und die vorliegende empirische Evidenz hierzu geprüft. In Abschnitt 3 wird ihre Relevanz für die deutsche Sozialversicherung untersucht. In Abschnitt 4 werden institutionelle Reformoptionen zur Bewältigung der aufgezeigten Problematik diskutiert, und Abschnitt 5 enthält eine kurze Schlussfolgerung.

\section{Zum Zusammenhang zwischen Leibrenten und Lebenserwartung}

\subsection{Theorie}

Aus welchen Marktversagensgründen ist überhaupt eine staatliche Rentenversicherung mit Zwangsmitgliedschaft zu empfehlen? Die Antwort auf diese

1. Gleichwohl lag der absolute Zuwachs in den 1990er Jahren ausgerechnet in dem Land mit 1,9 Jahren am höchsten, das bereits 1990 die höchste Lebenserwartung (nämlich 20,0 Jahre) aufwies, was belegt, dass eine etwaige Grenze noch lange nicht erreicht ist.

2. Es handelt sich um die Arbeiten von Davies und Kuhn (1992), Philipson und Becker (1998) und Breyer (1997). 


\section{Friedrich Breyer}

Frage in der Standard-Literatur lautet: weil auf Versicherungsmärkten asymmetrische Information über die Höhe des zu versichernden Risikos zwischen Nachfragern und Versicherungsunternehmen herrscht und daher nach dem Rothschild-Stiglitz-Modell in einem separierenden Gleichgewicht ein optimaler Versicherungsschutz allenfalls für die höchste Risikoklasse zu Stande kommen kann. Folgt man dieser Argumentation, so kann der Staat durch die Erzwingung der Teilnahme alle Risikoklassen besser stellen (Eckstein, Eichenbaum und Peled, 1985).

Auf den Leibrenten-Markt übertragen, lautet das Argument: da der einzelne über seine Lebenserwartung besser Bescheid weiß als ein privates Versicherungsunternehmen, werden auf einem Wettbewerbsmarkt Leibrenten nur $\mathrm{zu}$ sehr schlechten Konditionen angeboten und daher auch nicht gekauft. Diese Schlussfolgerung wird allerdings in der Realität nur zum Teil bestätigt. Eine gerade in letzter Zeit enorm wachsende Literatur über die Renditen auf Leibrenten-Märkten kommt im wesentlichen zu zwei Schlussfolgerungen (vgl. etwa James und Vittas, 1999, Cannon und Tonks, 2002, Finkelstein und Poterba, 2002):

1. Der Erwartungswert der Leistungsansprüche liegt, mit einem geeigneten Marktzins abgezinst, nur um 0-20 Prozent unter der Versicherungsprämie, wobei die „Kosten“ des Versicherungsschutzes geringer sind als in manchen anderen Versicherungssparten.

2. Der genannte Erwartungswert ist höher, wenn man die Zusammensetzung der tatsächlichen Käufer der Leibrenten zu Grunde legt, als wenn man Sterbetafeln der allgemeinen Bevölkerung zur Berechnung heranzieht.

Vor allem die zweite Beobachtung lässt den Schluss zu, dass es trotz tatsächlicher empirischer Relevanz der Antiselektions-Annahme nicht zu einem Zusammenbruch dieses Marktes gekommen ist - und das obwohl in vielen Ländern allein schon der Umfang staatlicher Rentenprogramme in einem erheblichem Maße zu einer Verdrängung dieser privaten Verträge geführt hat.

In den zu Grunde liegenden Standard-Modellen wird nun übereinstimmend angenommen, dass die Höhe des Risikos für das betroffene Individuum exogen ist, dass m.a.W. keine Möglichkeit besteht, auf die eigene Lebenserwartung einzuwirken. Dies ist der Ansatzpunkt der Arbeiten von Davies und Kuhn (1992) und Philipson und Becker (1998), ${ }^{3}$ die darauf hinweisen, dass der einzelne durch eine Vielzahl von Maßnahmen (Berufswahl, Ernährung, Rauchen, Bewegung, Inanspruchnahme medizinischer Leistungen) auf seine erwartete Lebenslänge Einfluss nehmen kann.

Um die Probleme analytisch auseinander zu halten, beginnen die Autoren mit einer Modellvariante, bei der sie vom Versicherungsproblem abstrahieren, indem sie die Lebensdauer als deterministisch, aber von der eigenen Aktivität

3. Interessanterweise wird die zuerst erschienene Arbeit von den Autoren der anderen nicht zitiert, obwohl sie im Journal of Public Economics erschienen war. 


\section{Auf Leben und Tod}

abhängig behandeln. In diesem Modell wird die Abwägung deutlich, vor der das repräsentative Individuum mit einem gegebenen Vermögen (z.B. in Form von Humankapital) in einer Welt ohne Sozialversicherung steht: Je mehr Ressourcen es in die Verlängerung der Lebensdauer investiert, um so weniger bleibt insgesamt für den Konsum übrig und um so mehr wird, weil dieser geringere Restbetrag auf eine größere Zeitspanne umgelegt werden muss, der Konsum pro Periode reduziert. Abgesehen von dem Extremfall, in dem die Lebensdauer von dem Individuum lexikographisch gegenüber dem Konsum (einschließlich der Freizeit) je Zeiteinheit bevorzugt wird, gibt es ein inneres Optimum, in dem nicht alle Möglichkeiten zur Lebensverlängerung ausgenutzt werden.

Dieses Kalkül wird nun zum einen durch die Existenz einer obligatorischen Krankenversicherung gestört: Indem der Preis der Lebensverlängerung durch medizinische Behandlung auf null reduziert wird, wird die individuell-rationale Nachfrage nach Lebenslänge nach oben verzerrt. Dies ist das bekannte Phänomen des Verhaltensrisikos (engl. „moral hazard“) in der Krankenversicherung (vgl. etwa Breyer, Zweifel und Kifmann, 2002, Kap. 6). Dieses hat jedoch nicht nur die bekannte statische, sondern auch eine dynamische Komponente: Da der einzelne aus der Krankenversicherung um so mehr Nutzen ziehen kann, je größer das medizinische Wissen ist, sind die Wähler auch als Steuerzahler bereit, Investitionen in die medizinische Forschung staatlich zu subventionieren, womit die technologischen Möglichkeiten zur Lebensverlängerung permanent vergrößert werden. ${ }^{4}$ Dabei spielt die Umlagefinanzierung in der gesetzlichen Krankenversicherung eine entscheidende Rolle: Da der Medianwähler noch den größten Teil der Leistungsansprüche vor sich, das Schwergewicht der Beitragszahlungen aber bereits hinter sich hat, hat er ein Interesse an der resultierenden Leistungsausweitung. ${ }^{5}$

Ein analoges Verhaltensrisiko ist jedoch auch mit der Existenz von (privaten oder staatlichen) Leibrenten-Versicherungen verbunden. Diese schützen zum einen besser gegen die finanziellen Folgen unerwarteter Langlebigkeit, als es die reine Ersparnis könnte, d.h. sie vermeiden sowohl Verarmung im sehr hohen Alter als auch ungeplante Vererbung. Andererseits verzerren sie jedoch die Abwägung zwischen optimaler Lebenslänge (bzw. -erwartung) und Konsum zugunsten der Lebenserwartung, solange die Höhe der monatlichen Leibrente nicht - was praktisch undurchführbar wäre - mit jeder lebensverlängernden Aktivität sofort in aktuarischer Manier nach unten angepasst wird.

Auf Märkten für private Leibrentenverträge wird das Problem im Idealfall (s. Abschnitt 4.1 für mögliche Abweichungen in der Praxis) so gelöst, dass die

4. Miller (2002) vertritt demgegenüber die These, dass heute wegen einer weit verbreiteten "Gerontophobie" sogar zu wenig in die Erforschung der Alterungsprozesse beim Menschen investiert wird. Nach seiner Auffassung könnten Fortschritte auf diesem Gebiet die Lebenserwartung des Menschen noch weit stärker ausdehnen als krankheitsspezifische Forschung.

5. Für die umlagefinanzierte Rentenversicherung ist dieser politisch-ökonomische Mechanismus zuerst von Browning (1975) entdeckt worden. 


\section{Friedrich Breyer}

angebotenen Tarife die aus der Versicherung erwachsenden Anreize zur Lebensverlängerung bereits berücksichtigen und damit eine entsprechende Relation zwischen Leibrente und Versicherungsprämie vorsehen. Die Individuen werden sich dem entsprechend mit Leibrentenverträgen abfinden, die keine volle Sicherung des Lebensstandards erlauben, und ihren Alterskonsum zum restlichen Teil doch aus „normaler“ Ersparnis decken. Denkbar wäre auch eine progressive Beziehung zwischen Leibrentenhöhe und Prämie, falls sich das Verhaltensrisiko nur bei hohen Leibrenten zeigt. Ein solcher Zusammenhang lässt sich allerdings nur dann praktisch durchsetzen, wenn die Individuen beim Kauf einer Leibrente alle bereits bestehenden Verträge offen legen müssen.

Anderes gilt für staatliche Rentensysteme mit Zwangsmitgliedschaft, die von vornherein nach dem Prinzip der Lebensstandardsicherung aufgebaut sind. Hier kommt das Verhaltensrisiko in vollem Umfang zur Geltung, da die Individuen mit einer auskömmlichen Versorgung bis ans Lebensende auf Kosten anderer - der nachfolgenden Generation - rechnen können und daher allen Anlass haben, dieses so weit wie möglich hinaus zu schieben. Damit verzerren diese Systeme das relevante Verhalten ihrer Mitglieder und führen zu einer „überoptimalen“ Investition in die Lebenslänge.

\subsection{Empirische Evidenz}

Wie steht es nun mit der empirischen Evidenz zu der oben dargestellten These, dass die Lebenserwartung gestiegen ist, weil es sich „lohnt“, länger zu leben? Hierzu legen Philipson und Becker (1998) eine Untersuchung mit Individualdaten von pensionierten Beamten aus den USA vor und zeigen, dass deren Mortalitätsrate mit der Höhe der Leibrente abnimmt. Diese Evidenz ist allerdings nicht schlüssig, da der Zusammenhang ebenso gut auf der zu Grunde liegenden positiven Korrelation zwischen Einkommen (bzw. Bildungsgrad) und Lebenserwartung beruhen kann.

Um diesen Einfluss auszuschalten, untersuchen wir im folgenden Daten auf Länder-Basis, bei der die Heterogenität im Einkommen durch die Aggregation beseitigt ist. Angaben über den relativen Wohlstand von Rentnern in 18 OECD-Ländern (gemessen durch das Durchschnittseinkommen eines Rentnerhaushalts als Anteil am Durchschnittseinkommen eines Arbeitnehmerhaushalts) kann man einer Studie von Förster und Pellizzari (2000) entnehmen (vgl. Tabelle 1, rechte Spalten). Diese Angaben sind nach zwei Altersgruppen (65 bis 74 und ab 75 Jahren) differenziert. Es besteht allerdings eine enge Korrelation $(\mathrm{R}=0,737)$ zwischen den beiden Reihen, so dass es in einer Regressionsrechnung sinnvoll erscheint, nur jeweils eine dieser Variablen als erklärende Größe für die Rest-Lebenserwartung einzubeziehen. Als weitere Erklärungsgrößen beziehen wir das Pro-Kopf-Bruttoinlandsprodukt und den Anteil der Gesundheitsausgaben ein, die beide einen positiven Effekt auf die Restlebenserwartung haben sollten.

Die in Tabelle 2 präsentierten OLS-Schätzungen unterscheiden ferner zwischen der Restlebenserwartung von 65jährigen Männern und Frauen, 
Auf Leben und Tod

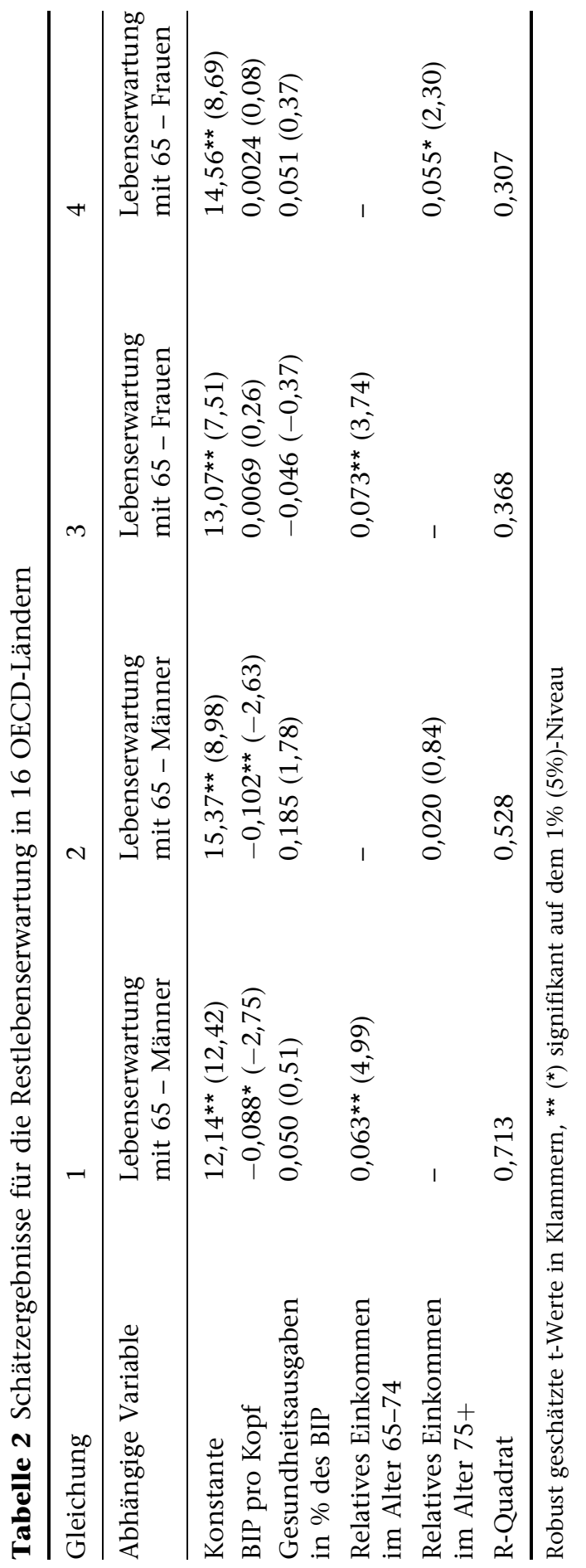




\section{Friedrich Breyer}

jeweils bezogen auf das Jahr 1999. In Klammern sind die robust geschätzten t-Werte angegeben, die mit Hilfe des White-Schätzers für Heteroskedastie berechnet wurden: ${ }^{6}$ Die Güte der Anpassung ist bei den Männern sehr viel höher als bei den Frauen, wobei von den beiden Maßen für den relativen Wohlstand das Einkommen der „jungen“ Alten eine größere Erklärungskraft besitzt: Danach ist eine Verbesserung der relativen Einkommensposition von Haushalten im Alter zwischen 65 und 74 Jahren um 10 Prozentpunkte mit einer Verlängerung der Restlebenserwartung um ca. 8 Monate verbunden, und der Effekt ist für beide Geschlechter auf dem 1\%-Niveau signifikant. Der geschätzte Effekt des relativen Einkommens älterer Rentner ist nur für Frauen ähnlich groß und auf dem 5\%-Niveau signifikant (Gleichung 4).

Überraschender Weise ist kein Einfluss der Gesundheitsausgaben auf die Lebenserwartung von 65jährigen festzustellen, und die Höhe des Pro-KopfProdukts wirkt sich bei Männern sogar signifikant negativ auf die Langlebigkeit aus. Dieses letzte Resultat überrascht nicht, wenn man jüngste Ergebnisse von Gerdtham und Ruhm (2002) betrachtet, die ebenfalls an OECD-Daten aus den vergangenen vier Jahrzehnten gezeigt haben, dass die Sterblichkeit insgesamt signifikant positiv auf die makroökonomische Prosperität (gemessen an einer niedrigen Arbeitslosenquote) reagiert. ${ }^{7}$ Was als für das einzelne Land im Konjunkturverlauf gilt, scheint sich auch im Querschnitt für unterschiedliche Niveaus des Pro-Kopf-Produkts zu bestätigen.

Natürlich sind die gefundenen Zusammenhänge zwischen der relativen Einkommensposition der Rentner und der Restlebenserwartung noch lange nicht als Beweis für die Gültigkeit der zuvor dargestellten Theorie zu werten, da die verfügbaren Daten doch sehr spärlich sind. Zudem sind alternative Erklärungen für den gefundenen Zusammenhang denkbar. So könnte sich der Wohlstand schon deshalb als lebensverlängernd erweisen, weil er ein gesünderes Leben ermöglicht. Ein weiterer Mangel der Daten ist darin zu sehen, dass sich die Einkommens-Variable ebenso wie die Rest-Lebenserwartung auf das selbe Jahr (1999) beziehen, während man theoretisch, wenn überhaupt, eine verzögerte Wirkung der Einkommensposition auf die Lebenserwartung vermuten würde.

Immerhin sind die Daten mit den Hypothesen von Davies und Kuhn sowie Philipson und Becker vereinbar. Dies ist um so bemerkenswerter, als man aus der Sicht der Budgetrestriktion einer staatlichen Rentenversicherung eher einen negativen Zusammenhang zwischen der Einkommensposition der Rentner und ihrer Restlebenserwartung vermuten würde: je länger die Rentner leben, eine desto geringere Rente kann man aus einem gegebenen Ausgabenvolumen finanzieren. Daher zeigt der gefundene positive Zusammenhang, dass die

6. Die Schätzgleichung bezieht sich auf 16 der 18 Länder, für die Einkommensdaten verfügbar sind. Ungarn und die Türkei wurden eliminiert, da sie von den restlichen Ländern in der Lebenserwartung stark nach unten abweichen.

7. Diese Autoren unterscheiden allerdings nicht, welches Geschlecht besonders von der erhöhten Sterblichkeit betroffen ist. 


\section{Auf Leben und Tod}

Rentner mit wachsender Lebenserwartung einen deutlich zunehmenden Anteil des Volkseinkommens absorbieren.

\section{Relevanz für das deutsche Rentensystem}

Die oben dargestellte Ineffizienz ist für die deutsche gesetzliche Rentenversicherung in vollem Umfang relevant. Zum einen orientiert sie sich vom Umfang der Rentenansprüche her am Prinzip der Lebensstandardsicherung, geht also zumindest in der Zielsetzung weit über eine reine Grundsicherung hinaus. Zudem sind die Rentenansprüche des einzelnen in keiner Weise an seinen Gesundheitszustand oder an andere Risikofaktoren gekoppelt, die eine Aussage über die individuelle Lebenserwartung ermöglichen würden.

In Breyer (1997) habe ich in erster Linie die Gerechtigkeit der Verteilung gegebener Gesamtmittel unter den Mitgliedern einer Alterskohorte thematisiert, die sich aus der gegenwärtig verwendeten Rentenformel ergibt. Nach dieser erhalten die „langlebigen“ Mitglieder einen größeren Anteil am Beitragsaufkommen der darauffolgenden Erwerbsgeneration als diejenigen, die früh sterben. Dabei beziehen sich die Begriffe „langlebig“ und „früh sterben“ nicht auf die ex-post realisierte Lebenslänge, sondern auf die an Hand bestehender Risikofaktoren bei Renteneintritt voraussagbare Lebenserwartung. Ungerechtigkeit in dieser Hinsicht betreffen z.B. die Behandlung von Angehörigen unterer Einkommens- und Bildungsschichten, die empirisch gesichert eine signifikant verringerte Lebenserwartung haben und deshalb nach der geltenden Rentenformel auch noch eine geringere Rendite auf ihre Beiträge zur Rentenversicherung erhalten, sowie Männer, deren erwartete Rentenlaufzeit um mehrere Jahre niedriger ist als die von Frauen.

$\mathrm{Ob}$ diese positive Korrelation zwischen Lebenserwartung und Nettobezügen aus der Rentenversicherung als "gerecht“ oder „ungerecht“ zu bewerten ist, hängt allerdings von der verwendeten Gerechtigkeitsnorm ab. So argumentiert Wagner (1994, S.44), dass das gegenwärtig praktizierte Rentenrecht ein Individuum bereits im Mutterleib (und zwar bevor das Geschlecht determiniert ist) dagegen versichert, mit einer hohen Lebenserwartung und damit einem hohen Transferbedarf geboren zu werden. Es ist allerdings fraglich, ob die Individuen hinter dem Schleier des Nichtwissens einen solchen „Risikoausgleich“ überhaupt wünschen würden. Immerhin impliziert dieser nämlich, dass ein negatives Ereignis der „Lotterie“ Lebenserwartung mit einem negativen Nettotransfer aus der Rentenversicherung verbunden ist, und vice versa. Berücksichtigt man, dass hohe Lebenserwartung in der Regel mit einem guten Gesundheitszustand im Alter zwischen 60 und 65 Jahren einhergeht und daher der höhere Konsumbedarf durch einen späteren Renteneintritt ausgeglichen werden kann, so erscheint der geschilderte „Risikoausgleich“ nicht wünschenswert.

In einer neueren Arbeit argumentiert nun Brown (2002), dass die weit verbreiteten obligatorischen Leibrenten mit einheitlichen, also nicht risikobezogenen Auszahlungen, trotz der impliziten Umverteilung von den „Kurzlebigen“ zu 


\section{Friedrich Breyer}

den „Langlebigen“ gegenüber der reinen Marktlösung Pareto-überlegen seien, da auch die Angehörigen der benachteiligten Gruppen (in den USA z.B. schwarze Männer ohne High-School-Abschluss) bei hinreichendem Grad an Risikoaversion durch die Annuitisierung nutzenmäßig besser gestellt werden, als wenn es keine Leibrenten gäbe. Dabei übersieht er allerdings, dass es tatsächlich funktionierende Leibrentenmärkte gibt (s.o., Abschnitt 2.1), so dass der relevante Referenzmaßstab ein privater Leibrentenvertrag mit einer individuell risikobezogenen Auszahlung sein muss. Bei diesem Vergleich schneidet die obligatorische Leibrente für die betrachteten Risikogruppen nicht mehr vorteilhaft ab.

Neben der intragenerativen ist jedoch auch die intergenerative Verteilung berührt, da ein Teil der demographisch bedingten Verschlechterung des Beitrags-Leistungs-Verhältnisses in der deutschen Rentenversicherung nicht auf den Rückgang der Fertilität zurück, sondern auf das fast ebenso spektakuläre Absinken der Mortalität, insbesondere in den Altersgruppen über 65 Jahren zurück geht. Nach der gegenwärtig praktizierten Rentenformel wird die Last jeder weiteren Veränderung dieser Größe ausschließlich von den Beitragszahlern getragen, mithin von der nachfolgenden Generation. Der von der christlichliberalen Regierung unter Minister Blüm in der „Rentenreform 1999“ eingeführte "demographische Faktor" hätte wenigstens die Hälfte der zukünftigen Zuwächse in der Lebenserwartung in eine Kürzung der monatlichen Renten umgesetzt. In der Rentenreform 2001 unter Minister Riester wurde zwar das Rentenniveau insgesamt abgesenkt, eine weitere unerwartete Zunahme der Lebenserwartung geht aber voll zu Lasten der Beitragszahler, also der nachfolgenden Generation.

Im Zentrum dieser Arbeit steht jedoch weniger die Frage der Gerechtigkeit als die Ineffizienz, die von der Gestaltung des Rentensystems ausgelöst wird und die in einem zu starken Anreiz besteht, das eigene Leben zu verlängern, weil man die Kosten des Lebensunterhalts anderen aufbürden kann. Dieser Effekt, der durch die Absenkung des Rentenniveaus in der GRV nach und nach geringer wird, ist durch die 2002 eingeführte „Riester-Rente“ wieder stabilisiert worden, auch wenn es sich hierbei um eine freiwillige, kapitalgedeckte Vorsorge in Form privater Verträge handelt. Die Verzerrung des individuellen Kalküls kommt dadurch zu Stande, dass der Staat die Prämien für diese Altersvorsorge unter der Bedingung bezuschusst, dass die Versicherungsleistung in Form einer Leibrente und nicht z.B. in einer einzigen Summe ausgezahlt wird. Dadurch verhindert die Riester-Rente, dass der Anteil des Gesamtvermögens, den die Betroffenen in Form von Leibrenten halten, und damit gleichzeitig die Anreize zum Überleben zurückgeht.

\section{Reformoptionen}

\subsection{Die Teil-Pauschalierung der Rentenansprüche}

In meiner bereits zitierten Arbeit (Breyer, 1997) habe ich eine Reformoption dargestellt, die zur Lösung sowohl des Gerechtigkeits- als auch des 


\section{Auf Leben und Tod}

Effizienzproblems einen Beitrag leisten könnte. Diese besteht in einer Zweiteilung der Auszahlungsform der Rentenansprüche:

1. Die monatliche Rentenzahlung wird für alle Versicherten auf einen einheitlichen Betrag in der Größenordnung der Sozialhilfe für andere NichtArbeitsfähige festgesetzt.

2. Sollten die nach heutigem Recht berechneten Rentenansprüche über den kapitalisierten Wert dieser "Grundrente“ hinaus gehen, so wird die Differenz an den Versicherten zu einem bestimmten Stichtag (z.B. bei Vollendung des 65. Lebensjahres) als Pauschalbetrag ausgezahlt.

Die monatliche Grundrente soll dabei verhindern, dass ein Versicherter vor dem Lebensende sein gesamtes Vermögen einschließlich der Pauschalrente ausgibt und dann mittellos dasteht - in der Hoffnung, dass die Gesellschaft ihn nicht verhungern oder erfrieren lässt.

Allerdings muss zusätzlich die Frage beantwortet werden, welche Lebenserwartung der Berechnung des Barwerts der „Grundrente“ zu Grunde gelegt werden soll. Hierzu gibt es mindestens drei Möglichkeiten:

a) die individuelle Rest-Lebenserwartung des Betroffenen,

b) die gruppenspezifische Lebenserwartung seiner sozioökonomischen Bezugsgruppe (definiert etwa durch Einkommen oder Bildungsabschluss), ${ }^{8}$

c) die Lebenserwartung der Gesamtbevölkerung.

Dabei ist klar, dass die erste Option am schwierigsten zu berechnen ist, aber dem mit der ausgezahlten Leibrente verbundenen Verhaltensrisiko am stärksten entgegen wirkt.

Risikoaverse Individuen, die an einer Verstetigung des Konsumstroms über ihre (unbekannte) Lebensdauer interessiert sind, könnten den Pauschalanteil ihres Rentenbezugs bei privaten Anbietern ebenfalls in eine monatliche Leibrente umwandeln. Diese werden die relative Höhe der Leibrenten den Unterschieden in der Lebenserwartung der Nachfrager anpassen. Dazu sind - analog zum Vorgehen bei Abschluss einer Lebensversicherung - Gesundheitstests erforderlich, mit denen der Versicherer auf den gleichen Informationsstand zu gelangen versucht wie der Versicherte selbst. Werden diese Tests nur oberflächlich ausgeführt, so resultieren sie in einer - gemessen am Wissen der Betroffenen - zu geringen Differenzierung der Rentenhöhen. Folglich werden die im versicherungstechnischen Sinne „guten Risiken“, d.h. jene Personen mit einer unterdurchschnittlichen Rest-Lebenserwartung, auf den Kauf einer Leibrente verzichten. Die daraus resultierende „negative Auslese" ist für den Versicherer jedoch verlustbringend. Folglich hat er allen Anreiz zur Sorgfalt bei der Risikoeinstufung.

8. Hierbei würde natürlich die politisch brisante Frage auftreten, ob das Geschlecht zur Gruppenabgrenzung verwendet werden sollte, wodurch dieser Barwert bei Frauen cet. par. höher ausfiele als bei Männern. 


\section{Friedrich Breyer}

Es ist zu vermuten, dass nicht das gesamte Volumen der Pauschalkomponente in private Leibrenten umgewandelt wird, da ein Teil der Versicherten zu Beginn ihres Rentenalters - in Erwartung späterer gesundheitsbedingter Konsumeinschränkungen (Börsch-Supan und Stahl, 1991) - größere Konsumprojekte wie teure Reisen durchführen werden.

Kommt es dennoch auf breiter Front zu einer Umwandlung des Pauschalanteils in private Leibrenten, so bleibt das Verhaltensrisiko auch für diesen Teil in entsprechendem Umfang wirksam. Die langfristige Folge einer Unterschätzung dieses Effekts durch die Versicherer könnten Verluste aus den Leibrenten-Verträgen sein, die die Versicherungsunternehmen in Zahlungsschwierigkeiten bringen könnten. Es bleibt dann abzuwarten, wie diese mit den Verlusten umgehen, wobei verschiedene Reaktionen denkbar sind:

a) Die Aktionäre der Versicherungsunternehmen könnten den Kapitalverlust akzeptieren,

b) Die Versicherungsunternehmen könnten in den Konkurs gehen und damit die Erfüllung der Verträge verweigern.

c) Die Versicherungsunternehmen könnten ihre Kunden vor die Wahl stellen, eine prozentuale Leistungskürzung zu akzeptieren, um den Konkurs abzuwenden,

d) die Unternehmen könnten ihre Zahlungsverpflichtungen aus den laufenden Beitragseingängen nachfolgender Versichertenkohorten erfüllen.

Strategie c) würde einer Umlage innerhalb der einzelnen Versichertenkohorte entsprechen, Strategie d) jedoch einer Umlage mit intergenerativem Transfer. Falls der Gesetzgeber diese Strategie zulässt oder sogar unterstützt, würde die Aufspaltung in Sozialhilfe-Rente und Pauschalbetrag allerdings wirkungslos. Dass eine solche Gefahr nicht nur theoretischer Natur ist, zeigt die Erfahrung mit der Privaten Krankenversicherung in Deutschland, die ebenfalls für die durch medizinischen Fortschritt ausgelösten Ausgabensteigerungen im Alter keine ausreichenden Rückstellungen gebildet hatte und seit einigen Jahren gesetzlich durch das Versicherungsaufsichtsgesetz sogar gezwungen - ein Umlageverfahren mit Quersubventionierung der Tarife der älteren durch die Beiträge der jungen Versicherten betreibt.

\subsection{Flankierende Maßnahmen}

Nicht nur die Rentenversicherung, sondern auch die ebenfalls mit Zwangsbeiträgen und nach dem Umlageverfahren finanzierte Krankenversicherung wird in den kommenden Jahrzehnten eine erhebliche demographische Last tragen müssen. Hier kommt jedoch hinzu, dass in Folge des technischen Fortschritts in der Medizin, der sich in erster Linie in neu entwickelten Behandlungsformen für zuvor unheilbare Krankheiten äußert, der Ausgabentrend besonders stark steigend ist (Breyer und Ulrich, 2000). 


\section{Auf Leben und Tod}

Die immer größer werdende Kluft zwischen dem technisch Machbaren und der medizinischen Versorgung, die mit einem gleich bleibendem Anteil des Sozialprodukts finanzierbar ist, führt schon heute zu erheblichen Spannungen zwischen den politisch Verantwortlichen und den Leistungserbringern im Gesundheitswesen. Die bislang politisch verfolgte Strategie einer Budgetierung der Ausgaben stößt mehr und mehr an ihre Grenzen, da sie zur impliziten Rationierung von Gesundheitsleistungen führt, d.h. medizinisch sinnvolle und im Prinzip im Leistungskatalog der gesetzlichen Krankenversicherung enthaltene Leistungen werden im Einzelfall aus Kapazitätsgründen nicht erbracht. Dass dieser Zustand heute schon erreicht ist, dafür gibt es zahlreiche empirische Belege (z.B. Kuhlmann, 1998, Schultheiss, 2001). Mit einem Rechtsstaat ist ein solcher Zustand der Rechtsunsicherheit allerdings nur schwer vereinbar, und es wird daher in letzter Zeit verstärkt über die Notwendigkeit eines Übergangs zu expliziter Rationierung, d.h. einer Verkürzung des Leistungskatalogs der gesetzlichen Versicherung nachgedacht (Breyer und Schultheiss, 2001, Stiftung Marktwirtschaft, 2002).

Bedenkt man, dass ein erheblicher Teil der medizinischen Versorgung nicht zur Erhöhung der Lebensqualität, sondern zur Verlängerung der Lebensdauer aufgewendet wird - man denke etwa an Organtransplantationen -, so sieht man, dass die Kollektivfinanzierung dieser Leistungen, wie von Philipson und Becker zu Recht festgestellt, die Ineffizienz in Form einer „Überinvestition“ in die Lebensdauer verschärft. Diese Überlegungen legen es nahe, lebensverlängernde Maßnahmen der Hochtechnologie-Medizin für sehr alte Patienten als potenzielle Kandidaten für eine Entfernung aus dem Leistungskatalog des kollektiv finanzierten Gesundheitswesens zu diskutieren. Nach einer angemessenen Übergangsfrist müssten die Menschen mit einer besonders intensiven Präferenz für die Lebenslänge diese Leistungen dann entweder durch eine private Zusatzversicherung abdecken oder aus eigener Tasche bezahlen. Entgegen einer landläufigen Meinung würde eine derartige altersbezogene Rationierung von Gesundheitsleistungen die Gleichheit der Bürger in einer fundamentalen Hinsicht (nämlich bezüglich der Chance, ein bestimmtes Lebensalter zu erreichen) nicht verringern, sondern im Gegenteil sogar steigern (Breyer und Schultheiss, 2002).

\section{Schlussbemerkungen}

Die Menschen in den hoch entwickelten Industriestaaten werden immer älter. Manche Wissenschaftler sehen darin bereits erste Schritte zur Verwirklichung eines Traums, den die Menschheit schon immer hatte, nämlich die eigene Sterblichkeit aufzuheben (Bernholz, 2002). Unabhängig davon, wie man sich zu diesem Wunsch auf individueller und kollektiver Ebene stellt, die sich abzeichnenden Schritte zu seiner Verwirklichung stellen unsere sozialen Sicherungssysteme auf eine Zerreißprobe, die diese nicht bestehen werden. 


\section{Friedrich Breyer}

Umgekehrt leisten eben diese Sozialsysteme der Illusion Vorschub, der Mensch könne seine Lebensdauer ständig ausdehnen, ohne die dadurch entstehenden Kosten in Form vermehrter Arbeit und verringerten Konsums tragen zu müssen. Sie verstoßen damit eklatant gegen das „Prinzip der vollständigen Internalisierung“ (Breyer und Kolmar, 2001, Kap. 3), nach dem eine effiziente Allokation nur erreicht werden kann, dass jeder an der Grenze alle Kosten und Erträge seiner Handlungen trägt.

In diesem Beitrag wurden konkrete Reformoptionen vorgestellt, mit denen die dargestellten Fehlanreize beseitigt werden könnten. Aus heutiger Sicht mögen sie manchem Leser als revolutionär und abwegig erscheinen. Ich bin aber überzeugt, dass Reformen in dieser Richtung erforderlich sind, um das Niveau der sozialen Sicherung und gleichzeitig die Freiheitlichkeit der Wirtschaftsordnung langfristig zu garantieren.

\section{Literaturverzeichnis}

Bernholz. P. (2002), The Revolt of Promethean Man, Homo Oeconomicus 18, 437-450. Börsch-Supan, A. und Stahl, K. (1991), Life-Cycle Savings and Consumption Constraints, Journal of Population Economics 4, 233-255.

Breyer, F. (1997), Sind „äquivalente“ Renten fair?, in: R. Hauser (Hrsg.), Reform des Sozialstaats I, Duncker \& Humblot, Berlin, 169-180.

Breyer, F. und Kolmar, M. Grundlagen der Wirtschaftspolitik, Mohr, Tübingen.

Breyer, F. und Schultheiss, C. (2001) On the Ethics of Primary Rationing in Health Care, Associations (Journal for Legal and Social Theory) 5, 195-211.

Breyer, F. und Schultheiss, C. (2002), 'Primary' Rationing of Health Services in Ageing Societies: a Normative Analysis, International Journal of Health Care Finance and Economics 2, 247-264.

Breyer, F. und Ulrich, V. (2000), Gesundheitsausgaben, Alter und medizinischer Fortschritt: eine Regressionsanalyse, Jahrbücher für Nationalökonomie und Statistik 220, $1-17$.

Breyer, F., Zweifel, P. und Kifmann, M. (2002), Gesundheitsökonomie, 4. Aufl., SpringerVerlag, Berlin u.a.

Brown, J.R. (2002), Redistribution and Insurance: Mandatory Annuitization with Mortality Heterogeneity, NBER Working Paper No. 9256, October.

Browning, E.K. (1975), Why the Social Insurance Budget is too Large in a Democracy, Economic Inquiry 13, 373-388.

Cannon, E. und Tonks, I. (2002), Annuity Prices, Money's Worth and Replacement Ratios: UK Experience 1972-2002, CMPO Working Paper No. 02/051, University of Bristol, September.

Davies, J.B. und Kuhn, P. (1992), Social Security, Longevity, and Moral Hazard, Journal of Public Economics 49, 91-106.

Eckstein, Z., Eichenbaum, M. und Peled, D. (1985), Uncertain Lifetimes and the Welfare Enhancing Properties of Annuity Markets and Social Security, Journal of Public Economics 26, 303-326.

Finkelstein, A. und Poterba, J. (2002), Selection Effects in the United Kingdom Annuities Market, Economic Journal 112, 28-50. 


\section{Auf Leben und Tod}

Förster, M.F. und Pellizzari, M. (2000), Trends and Driving Factors in Income Distribution and Poverty in the OECD Area, Labour Market and Social Policy Occasional Paper No. 42, OECD, Paris.

Gerdtham, U. und Ruhm, C.J. (2002), Deaths Rise in Good Economic Times, NBER Working Paper No. 9357, November.

James, E. und Vittas, D. (1999), Annuities Markets in Comparative Perspective: Do Consumers Get Their Money's Worth? Unpubl. Manuscript, The World Bank, September.

Kuhlmann, E. (1998), Zwischen zwei Mahlsteinen. Ergebnisse einer empirischen Studie zur Verteilung knapper medizinischer Ressourcen in ausgewählten klinischen Settings, in: G. Feuerstein und E. Kuhlmann (Hrsg.), Rationierung im Gesundheitswesen, Ullstein Medical, Wiesbaden, 11-80.

Miller, R.A. (2002), Extending Life: Scientific Prospects and Political Obstacles, Milbank Quarterly 80, 155-174.

Philipson, T.J. und Becker, G.S. (1998), Old-Age Longevity and Mortality-Contingent Claims, Journal of Political Economy 106, 551-573.

Schultheiss, C. (2001), Im Räderwerk der impliziten Rationierung - Eine Umfrage zu den Auswirkungen der Kostendämpfung im Gesundheitswesen, unveröff. Manuskript, Universität Konstanz, September.

Stiftung Marktwirtschaft, Hg. (2002), Mehr Eigenverantwortung und Wettbewerb im Gesundheitswesen, Berlin.

Wagner, G. (1994), Möglichkeiten des „Sozialversicherungsstaates“ - Prinzipielles und Reformvorschläge an den Beispielen der Gesundheits- und Umweltvorsorge, in: B. Riedmüller und T. Olk (Hrsg.), Grenzen des Sozialversicherungsstaates, Westdeutscher Verlag, Opladen, 37-56.

Abstract: Rapidly increasing life expectancy is seen by economists as a serious impediment to the financing of social security systems, no matter whether they are funded or not. However, what is less obvious is that these systems themselves may contribute to the increase in life expectancy by distorting people's incentives to invest in the length of their lives. This paper discusses theories of this moral hazard effect and offers some preliminary evidence on the empirical validity of this theory. I also present an option of reforming traditional social security systems which is designed to reduce this moral hazard effect. 
